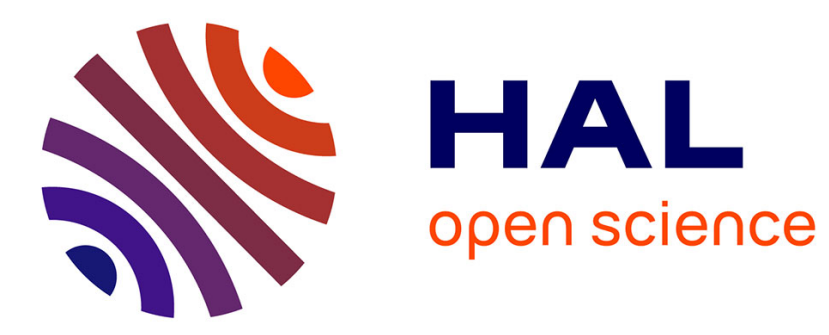

\title{
Dynamics of photon fluid flows driven by optical pistons
}

Abdelkrim Bendahmane, Gang Xu, Matteo Conforti, Alexandre Kudlinski, Arnaud Mussot, Sefano Trillo

\section{To cite this version:}

Abdelkrim Bendahmane, Gang Xu, Matteo Conforti, Alexandre Kudlinski, Arnaud Mussot, et al.. Dynamics of photon fluid flows driven by optical pistons. CLEO: QELS_Fundamental Science, May 2019, San Jose, United States. pp.FTh3B.5, 10.1364/CLEO_QELS.2019.FTh3B.5 . hal-02392665

\section{HAL Id: hal-02392665 \\ https://hal.science/hal-02392665}

Submitted on 4 Dec 2019

HAL is a multi-disciplinary open access archive for the deposit and dissemination of scientific research documents, whether they are published or not. The documents may come from teaching and research institutions in France or abroad, or from public or private research centers.
L'archive ouverte pluridisciplinaire HAL, est destinée au dépôt et à la diffusion de documents scientifiques de niveau recherche, publiés ou non, émanant des établissements d'enseignement et de recherche français ou étrangers, des laboratoires publics ou privés. 


\title{
Dynamics of photon fluid flows driven by optical pistons
}

\author{
Abdelkrim Bendahmane, ${ }^{1, *}$, Gang $\mathbf{X u}^{1, *}$, Matteo Conforti ${ }^{1}$, , Alexandre Kudlinski ${ }^{1}$, Arnaud Mussot ${ }^{1}$, \\ Sefano Trillo ${ }^{2}$ \\ ${ }^{1}$ Univ. Lille, CNRS, UMR 8523-PhLAM-Physique des Lasers Atomes et Mol'ecules, F-59000 Lille, France \\ ${ }^{2}$ Department of Engineering, University of Ferrara, Via Saragat 1, 44122 Ferrara, Italy \\ *These authors contributed equally to this work \\ abdelkrim.bendahmane@univ-lille1.fr
}

\begin{abstract}
We investigate the optical analogous of the piston shock problem in gas dynamics. Using fast temporal measurements, we recorded dispersive shock waves formed by the propagation of a bi-chromatic photon fluid along an optical fiber.

OCIS codes: (190.4370) Nonlinear optics fibers, (190.5530) Pulse propagation and temporal solitons.
\end{abstract}

\section{Introduction}

The piston problem, i.e. the dynamics in a uniform gas at rest under the action of a moving piston is fundamental problem of physics and a case study in shock wave physics [1]. Shock waves have been thoroughly investigated in many different branches of physics [2]. In conservative systems, shock waves are regularized by the formation, owing to dispersion, of rapidly oscillating non-stationary structures, called dispersive shock waves (DSWs) [3-5].

In this work, we investigate the analogous problem for a photon fluid, which turns out to be strongly influenced by the dispersive character of the medium. Our experiments make use of a fiber optics setup where an all-optically controlled quasi instantaneous discontinuity of frequency of the input light mimics the piston action. This configuration is a specific case of the general Riemann problem that concern propagation with discontinuities [6,7].

\section{Analogy with the piston problem and experimental setup}

More precisely, to mimic gas compression, we study the propagation, along a highly normal dispersive optical fiber, of a chirped square pulse with an abrupt jump of instant frequency (velocity) at its center (as illustrated in Fig. 1(a)). During the propagation of this dual-frequency pulse, the two parts propagate at different velocities mimicking gas compression. Indeed, the fast pulse part plays the role of a moving piston while the other one (the slow part) plays the role of the compressed gas. The internal collision and squeezing of these two parts eventually lead to the generation of a pair of DSWs connected by an intermediate plateau of constant density (Fig. 1(b)).

In order to generate the suitable bi-velocity square pulse needed to study the genuine Riemann problem, we temporally couple a square shaped pulse and a strong external M-shaped pulse. Both of these pulses are obtained by a direct intensity modulation of $\mathrm{CW}$ waves (Fig. 1(c)). The role of this M-shaped pulse is to induce a frequency shift on the square pulse via cross phase modulation (XPM) while propagating along $500 \mathrm{~m}$ of highly nonlinear fiber (HNLF). Since the linear slope of the M-shaped pulse abruptly change at its center, this allows to induce a quick shift in frequency at the center of the square pulse. Hence, synthesizing the desired state.

At the output of the HNLF fiber, the M-shaped pulse is spectrally filtered and the chirped square pulse is then injected into $15-\mathrm{Km}$ of dispersion compensating fiber (DCF) (fiber parameters are given in the caption of Fig. 1). The output light is characterized using an optical sampling oscilloscope (OSO).
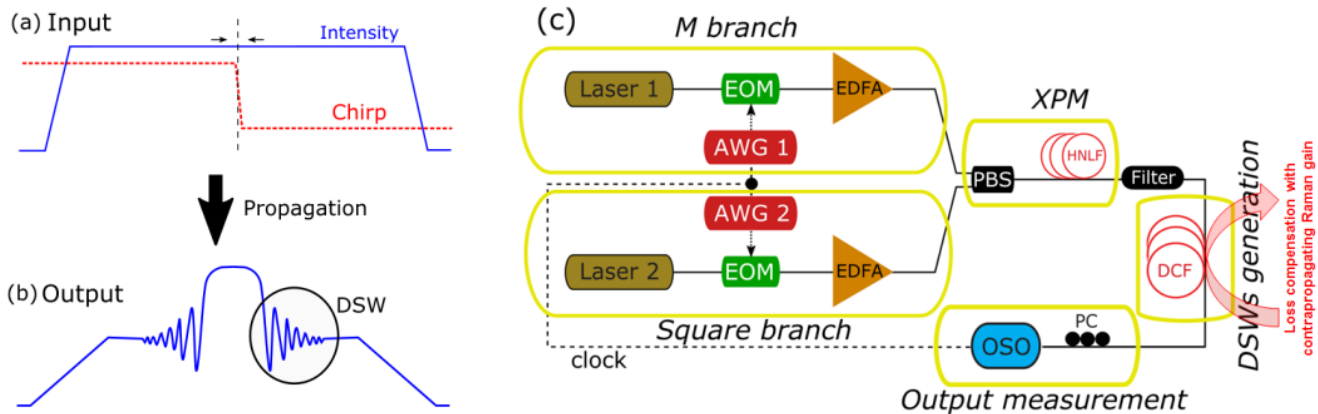

Fig. 1. (a,b) Illustration of the chirped square pulse and the generation of DSWs. (c) Experimental setup. AWG, Arbitrary Waveform Generator; EOM, electro-optic modulator; EDFA, erbium-doped fiber amplifier; PBS, polarization beam splitter; PC, Polarization controller; OSO, optical sampling oscilloscope; HNLF (length $500 \mathrm{~m}$; loss $\alpha=0.6 \mathrm{~dB} / \mathrm{km}$; zero dispersion wavelength ZDW $=1551.4 \mathrm{~nm}$; third order dispersion $\beta_{3}=0.53 \times 10^{-40} \mathrm{~s}^{3} / \mathrm{m}$; nonlinear coefficient $\left.\gamma=12 \mathrm{~W}^{-1} \mathrm{~km}^{-1}\right)$. DCF(length $15 \mathrm{~km} ; \alpha=0.5 \mathrm{~dB} / \mathrm{km} ; \beta_{2}=1.7 \times 10^{-25} \mathrm{~s}^{2} / \mathrm{m} ; \gamma=3 \mathrm{~W}^{-1} \mathrm{~km}^{-1}$ ); Initial condition of M pulse (peak power $7 \mathrm{~W}$, duration $2.4 \mathrm{~ns}$,

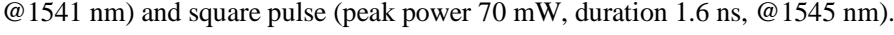




\section{Compressing Piston:}

We performed a series of experiments that demonstrate the generation of DSWs from the chirped pulse. Figure 2 (a) depicts the temporal profile of the $1.3 \mathrm{~ns}$ square shaped pulse recorded at the DCF input (HNLF output). The spectrogram in Fig. 2(b) provides us a clear description of the bi-chromatic properties of this pulse. This spectrogram was obtained by performing a heterodyne detection with the help of a tunable $\mathrm{CW}$ laser and a $70 \mathrm{GHz}$ photodiode. In the presented case, the total frequency shift induced by the M-shaped pump is $18.5 \mathrm{GHz}$.

Once injected into the DCF fiber, the square pulse undergoes nonlinear effect and we observed at the DCF output, for an input peak power of $210 \mathrm{~mW}$, the generation of a short central plateau, with several fast and regular oscillations aside (Fig. 2(c)). These oscillations correspond to a regularization of the shock and the formation of a pair of DSW [2]. More interesting, when we reduce the input peak power to almost $8 \mathrm{~mW}$, the pair of DSWs becomes connected by a periodic structure instead of a flat plateau (Fig. 2(d)), we also observe the apparition of vacuum points (points with no photon density). This special case has no equivalent in gas dynamics [7].

The propagation of the chirped square pulse in the DCF was also numerically simulated by integrating the generalized nonlinear Schrodinger equation taking into account our experimental conditions. Our simulations show a good qualitative agreement with observations (not represented here).
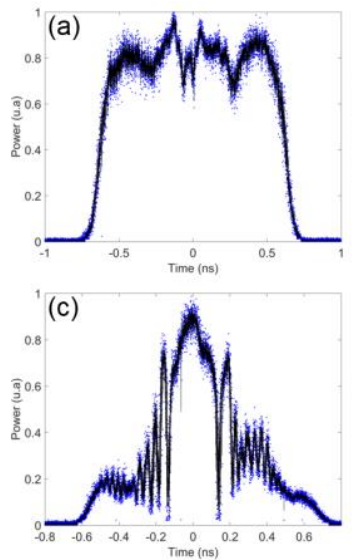

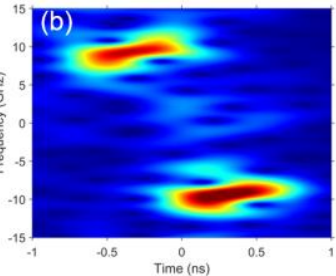

(d)

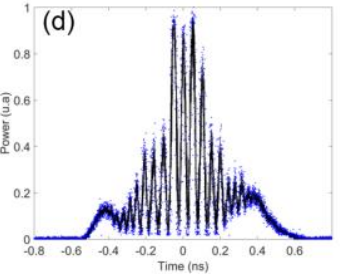

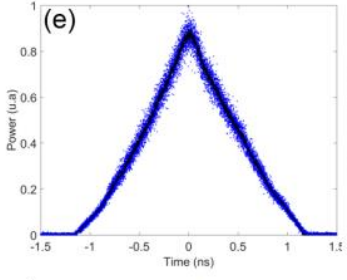

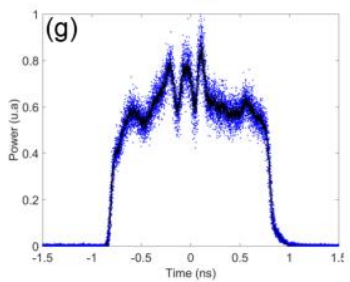

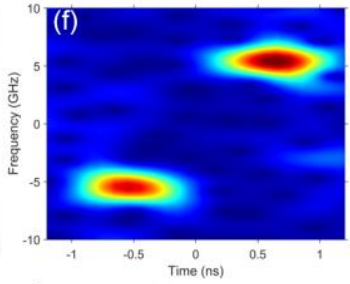

${ }^{1}$ (h)

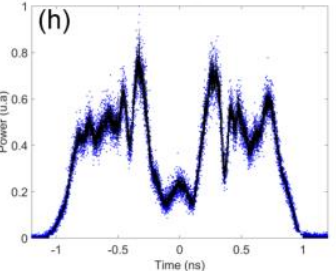

Fig. 2. (a-d) Piston configuration. (a) Intensity profile of the square pulse recorded at the DCF input. (b) Associated Spectrogram. (c,d) Intensity profile recorded at the DCF output for an input peak power of (c) $210 \mathrm{~mW}$ and (d) $8 \mathrm{~mW}$. (e-h) Anti-piston configuration. (e) Intensity profile of the triangle shaped pulse used to induce the chirp. Initial condition of the triangle pulse (peak power $5 \mathrm{~W}$, duration $2.4 \mathrm{ns,} \mathrm{@1536} \mathrm{nm).} \mathrm{(f)} \mathrm{Spectrogram} \mathrm{associated} \mathrm{to} \mathrm{the} \mathrm{input} \mathrm{square} \mathrm{pulse} \mathrm{(induced}$ frequency shift $=10.5 \mathrm{GHz})$. $(\mathrm{g}, \mathrm{h})$ Intensity profile recorded at the $(\mathrm{g}) \mathrm{DCF}$ input $(\mathrm{h})$ DCF output. (input peak power $=35 \mathrm{~mW}$ ).

\section{Retracting Piston:}

In a second set of experiments, we replaced the M-shaped pump by a triangle shaped one (Fig. 2(e)), which reverses the sign of the velocity profile compared to the previous case (see spectrogram in Fig. 2(f)). Instead of compressing each other, the two parts composing the input pulse ((Fig. 2(g)) undergo a decompression mechanism leading to the formation of a rarefaction waves pair, connected by a small finite plateau (as represented in Fig. 2(h)). This process is analogous to the gas dynamics case when the piston decompresses and exhausts the gas, leading to a low pressure inside a reservoir. Here also, numerical simulations agreed well with experiments (not shown here).

\section{Conclusion}

We studied the optical analogy of a gas compressed/decompressed by a piston. Our experiments consist in the propagation of a bi-chromatic photon fluid along a highly dispersive optical fiber. We observed generation of dispersive shock waves and rarefaction waves. These results are also well supported by numerical simulations and pave the way to study gas dynamics in relatively accessible optical fiber experiments.

\section{References}

[1] M. Hoefer et al., "Piston Dispersive shock wave problem", Physical Review Letters, 100, 084504(2008).

[2] G.B. Whitham, Linear and nonlinear waves (Wiley, New York, 1974).

[3] Z. Dutton et al., "Observation of quantum shock waves created with ultra-compressed slow light pulses in a Bose-Einstein condensate".

Science, 293, 663 (2001).

[4] J. Joseph et al., "Observation of shock waves in a strongly interacting fermi gas". Phys. Rev. Lett., 106, 150401 (2011).

[5] J. Fatome et al., "Observation of optical undular bores in multiple four-wave mixing". Phys. Rev. X., 4, 021022 (2014).

[6] G. Xu et al. "Dispersive dam-break flow of photon fluid", Phys. Rev. Lett., 118, 254101(2017).

[7] G. El et al. "Decay of an initial discontinuity in the defocusing NLS hydrodynamics, Physica D. 87, 186 (1995). 